RUNNING HEAD: SENSATION-SEEKING AND RISK

Within-Person Variability in Sensation-Seeking During Daily Life: Positive Associations with Alcohol Use and Self-Defined Risky Behaviors

David M. Lydon-Staley' ${ }^{1}$ Emily B. Falk ${ }^{2,4,4}$, Danielle S. Bassett ${ }^{15,6,77^{*}}$

'Department of Bioengineering, School of Engineering \& Applied Science, University of

Pennsylvania, Philadelphia, PA 19104 USA

${ }^{2}$ Annenberg School for Communication, University of Pennsylvania, Philadelphia, PA 19104

USA

${ }^{3}$ Department of Psychology, University of Pennsylvania, Philadelphia, PA 19104 USA ${ }^{4}$ Wharton Marketing Department, University of Pennsylvania, Philadelphia, PA 19104 USA sDepartment of

Electrical \& Systems Engineering, School of Engineering \& Applied Science, University of Pennsylvania, Philadelphia, PA 19104 USA

${ }^{6}$ Department of Neurology, Perelman School of Medicine, University of Pennsylvania, Philadelphia, PA 19104 USA

'Department of Physics \& Astronomy, College of Arts \& Sciences, University of Pennsylvania, Philadelphia, PA 19104 USA

${ }^{\circledR}$ Department of Psychiatry, Perelman School of Medicine, University of Pennsylvania, Philadelphia, PA 19104 USA

The most recent version of this manuscript has been accepted for publication at Psychology of Addictive Behaviors

*Corresponding author: Danielle S. Bassett, 210 S. 33 ${ }^{\text {rd }}$ Street, 240 Skirkanich Hall, Philadelphia, PA 19104-6321. Email: dsb@ seas.upenn.edu 


\begin{abstract}
Sensation-seeking is the seeking of varied, novel, and intense experiences and the willingness to take risks in order to engage in these experiences. Sensation-seeking is robustly associated with engagement in risky behaviors but important questions remain concerning the role of withinperson variability in sensation-seeking. We use data from a 21-day daily diary protocol from 167 participants (mean age $=25.37, S D=7.34)$ to test day-to-day, within-person associations between sensation-seeking and both alcohol use and self-reported risk-taking. Participants also reported the riskiest behavior they engaged in each day, allowing insight into the types of risks that participants take during the course of daily life. Multilevel model results indicate that days of higher than usual sensation-seeking are more likely to be days on which alcohol is consumed relative to days of no alcohol use, and that risk-taking is higher than usual on days of higher than usual sensation-seeking. Coupling natural language processing with network science tools, we reduce 2490 self-reports of the day's riskiest behavior to 20 communities reflecting a wide range of risk domains, including social, school, work, and drug use risks. Creating a risk-taking diversity score based on the identified domains of participant-elicited risk behaviors, we find that trait sensation-seeking is positively associated with greater diversity in the types of risks reported. In sum, we capture day-to-day fluctuations in sensation-seeking, observe that sensation-seeking and both alcohol use and risky behaviors are associated at the within-person level, and provide insight into the types of risks taken during the course of daily life.
\end{abstract}

Keywords: sensation-seeking; impulsivity; alcohol; risk; daily diary 


\section{Within-Person Variability in Sensation-Seeking During Daily Life: Positive Associations with Alcohol Use and Self-Defined Risky Behaviors}

Sensation-seeking is defined as the "seeking of varied, novel, complex, and intense sensations and experiences, and the willingness to take physical, social, legal, and financial risks for the sake of such experiences" (Zuckerman, 1994, p.26). Sensation-seeking as a trait is associated with engagement in behaviors typically deemed risky, including alcohol use (Hittner \& Swickert, 2006), cigarette-smoking (Lydon-Staley \& Geier, 2018), high risk or extreme sports (Jack \& Ronan, 1998), and high-risk sexual activities (Donohew et al., 2000). Sensation-seeking exhibits intraindividual change - changes construed as developmental, relatively enduring, and that occur over the course of years (Nesselroade, 1991; Ram \& Gerstorf, 2009). Especially marked declines in sensation-seeking occur over the course of adolescence and into young adulthood (Lynne-Landsman et al., 2011; Steinberg et al., 2018). In contrast to considerations of intraindividual change over longer time horizons, much less research examines intraindividual variability in sensation-seeking - changes that are relatively short-term, occur more rapidly than those falling under the purview of longitudinal intraindividual change, and that are construed as relatively reversible changes in comparison to intraindividual change (Nesselroade, 1991). Yet, sensation-seeking tendencies may vary within-person at short timescales, from day to day or hour to hour. Indeed, early in the development of the concept of sensation-seeking, state sensation-seeking scales were constructed with the hypothesis that the state preceding behavior would be more predictive of behavior than trait sensation-seeking (Neary, 1975; Zuckerman, 1994). This potential short-term variability has received little attention despite its potential importance for understanding engagement in risky behavior (Lydon-Staley \& Bassett, 2018). Here we provide an overview of sensation-seeking's purported role in risky behavior before 
making use of daily reports of sensation-seeking, alcohol use, and risk-taking to test the withinperson association among sensation-seeking and both alcohol use and other self-defined risky behaviors.

A substantial body of work has tested the contribution of sensation-seeking to risky behavior (Coskunpinar et al., 2013; Cyders et al., 2009; Miller et al., 2003). These studies provide insight into who may be most at risk for engaging in risk behaviors. Studies of intraindividual change in sensation-seeking provide insight into when during development individuals high in sensation-seeking may be most likely to engage in risk behaviors (Ashenhurst t al., 2015; Lydon-Staley \& Geier, 2018; Quinn \& Harden, 2013). Neither type of assessment, however, allows a test of the shorter-time scale within-person processes put forward by perspectives suggesting that risky behavior is preceded by moments of increased sensationseeking (Lydon-Staley \& Bassett, 2018; Neary, 1975; Zuckerman, 1994). For such a test we must turn to intensive repeated measures. These study designs repeatedly assess individuals as they go about their daily lives. The proximal (in place and time) nature of the assessment provides ecological validity and minimizes retrospective biases introduced in questionnaires asking participants to recall and aggregate information about longer periods of time (e.g., previous 30 days; Schwarz, 2007). Most importantly, for the current aim, intensive repeated measures capture within-person fluctuations in the phenomena of interest and, once appropriately treated to allow both between-person (e.g., "who is most likely to take risks?”) and within-person (e.g., "when is an individual most likely to take risks"?) inferences (Curran \& Bauer, 2011; Molenaar, 2004), can provide insight into intraindividual variability.

The collection of intensive repeated measures, although not new (Csikszentmihalyi et al., 1977; Larson \& Csikszentmihalyi, 2014), is increasingly feasible due to advances in, and the 
ubiquity of, mobile communication technologies (Pew Research Center, 2017). Few studies have capitalized on this increased feasibility to capture momentary sensation-seeking during the course of daily life. One notable exception included a study that used potential indicators of sensation-seeking ("I have driven recklessly") during the development of a scale designed to assess momentary impulsivity in daily life (Tomko et al., 2014). Due to poor convergent validity between aggregated scores of the momentary impulsivity scale across a 28-day period and scores on a traditional, retrospective self-report sensation-seeking questionnaire, however, the sensation-seeking indicators were not retained in the final scale. In a second relevant study that examined facets of impulsivity during daily life, momentary reports of behaviors related to sensation-seeking (e.g., "Since the last beep, did something risky") were averaged across seven days. This average score showed positive associations with trait sensation seeking tendencies as assessed during a laboratory visit (Sperry et al., 2018), suggesting that these levels of analysis are related between subjects. Within-person associations between sensation-seeking and risky behavior, however, were not examined in these studies, limiting our ability to understand how within person fluctuations in sensation seeking relate to relevant behaviors. Further, difficulties in validly capturing sensation seeking during daily life remain. This difficulty, in part, stems from the need for short measurement instruments in intensive repeated measures designs in order to avoid overburdening participants. Indeed, the use of single-item scales is common in intensive repeated measures studies due to the effort required of participants to respond to many survey prompts over relatively short periods of time (Fisher, 2012). Thus, although attention has turned to the capture of sensation-seeking during daily life, much work remains to be done in order to quickly but validly capture sensation-seeking during daily life and to answer questions 
concerning the role of within-person variability in sensation-seeking for understanding risky behavior.

\section{The present study}

We extend understanding of the association between sensation-seeking and risky behavior in multiple ways. First, we use daily sensation-seeking scales that reliably capture within-person variability in sensation-seeking to test the within-person hypothesis that days of higher than usual sensation-seeking are days of higher than usual alcohol use. Second, by asking participants to rate the extent to which their behavior is risky, we test the within-person hypothesis that days of higher than usual sensation-seeking are days of higher than usual selfreported risk-taking. Third, risk is both fact-laden and value-laden, containing both objective and subjective components, such that there is a need for subjective judgment during risk-taking (Hansson et al., 2010; Redmill, 2002). By having participants describe their everyday risk behaviors, we provide insight into the types of risks that are undertaken during the course of everyday life, moving beyond definitions of risky behaviors that are common in the field (e.g., smoking, illicit drug use, eating unhealthy foods, driving recklessly, and drinking excessively; Reyna \& Huettel, 2014) to subjective, participant-elicited definitions.

Finally, although the main focus of the present manuscript is on daily, within-person associations between sensation-seeking and both alcohol use and risk-taking, we leverage the intensive longitudinal data to examine associations between trait sensation-seeking as traditionally assessed with a baseline questionnaire (Hoyle et al., 2002; Whiteside \& Lynam, 2001) and between-person differences in aggregate estimates of day-to-day risk behavior. Given that sensation-seeking is associated with the seeking of varied, novel, and intense sensations and 
experiences, we test the hypothesis that trait sensation-seeking is positively associated with a greater diversity in the types of risky behaviors that are practiced in daily life. To do this we couple natural language processing with network science tools to reduce the dimension of many (2490) idiosyncratic reports of risky behavior into twenty risk domains, providing an illustration of how the complexity of subjective risk as defined by participants themselves may be distilled into meaningful insights using existing analytic methods.

\section{MATERIALS AND METHODS}

We made use of data from the Knowledge Networks Over Time study, an intensive longitudinal study designed to provide insight into day-to-day intraindividual variability across a range of domains of functioning. The variables used in the present study have not been reported on previously (Lydon-Staley et al., 2018). All research was conducted in accordance with the institutional review board at our host university.

\section{Participants}

Participants were 167 individuals (136 female, 29 male, 2 other gender) recruited through poster, Facebook, Craigslist, and university research site advertisements in Philadelphia and the surrounding university community. Individuals were eligible if they met 4 criteria: 1) being between 18 and 65 years of age; 2) having consistent access to a home computer with internet; 3) being willing to complete 21 consecutive days of surveys; 4) being willing to visit the research laboratory for 1 hour. Participants were aged between 18.21 and 65.24 years of age $(M=25.37$, $S D=7.34)$, and identified as African American/Black (8.38\%), Asian (23.35\%), Hispanic/Latino (4.79\%), Multiracial (6.59\%), other (5.39\%), White (49.10\%), and missing information (2.40\%). Participants identified as bisexual (7.78\%), gay (4.19\%), heterosexual (79.04\%), lesbian 
(1.20\%), other (5.99\%), and missing information (1.80\%). Participants reported a yearly family income ranging from 'under $\$ 20,000$ ' to ' $\$ 200,000$ or more' (Modal income $=$ ' $\$ 20,000$ $\$ 49,000$ '). Participants' education spanned less than a high school degree $(0.60 \%)$, high school degree $(8.98 \%)$, associate's degree or some college but no degree $(30.54 \%)$, college degree $(37.72 \%)$, graduate or professional training $(20.96 \%)$, or missing information $(1.20 \%)$.

\section{Procedure}

Interested participants encountering study advertisements were directed to a website with study information and a consent form. After confirming that participants met inclusion criteria, participants were contacted via telephone with a description of the study and were offered an opportunity to assent or decline participation. If individuals assented, an email was sent with a baseline survey containing demographic questionnaires and additional scales that were not the focus of the present study. After the baseline survey, participants completed a laboratory session. At the laboratory session, participants filled out additional questionnaires, received training in the daily assessment protocol, and were guided through the installation of an app necessary for an internet browsing study component that we do not report on in the present study. Following the laboratory study, a 21-day diary assessment protocol consisting of two components was initiated. The first component was a daily diary composed of survey questionnaires that took approximately 5 minutes to complete. The second component came immediately after the daily diary component and was a 15 minute internet browsing task that we do not report on in the present manuscript. Links to the daily assessments were emailed to participants at 6:30 PM each evening. Participants requesting reminders received a text message at 6:40 PM to notify them that survey links had been emailed. Participants were instructed to complete the daily assessments before going to bed but were also informed that links remained open until 10:00 AM 
the next morning. In cases where participants completed the surveys the following morning, they were instructed to report as if they were completing the survey on the previous evening. Participants were compensated with gift cards to Amazon.com at each study phase: $\$ 25$ after completing the baseline assessment and the laboratory visit. For the daily assessment, completion was incentivized by making participant payment contingent on completion: completion of $3,4,5$, 6 , and 7 surveys each week was compensated with gift cards worth $\$ 10, \$ 15, \$ 20, \$ 25$, and $\$ 35$, respectively. Continued participation through the daily assessment was further incentivized by using a raffle for which an iPad mini as a prize. Completion of all 7 surveys each week resulted in one entry into the raffle drawing.

\section{Measures}

The present study made use of participants' reports of demographic and trait characteristics from the baseline surveys and their daily diary reports.

Day's Sensation-Seeking. Day's sensation-seeking was measured using 2 items adapted from the Fun-Seeking subscale of the BIS/BAS scales (Carver \& White, 1994) and the Excitement-Seeking subscale of the Revised Neuroticism, Extraversion, and Openness Personality Inventory (Costa \& McCrae, 1992): "How much of the time today did you crave excitement?" and "How much of the time today did you crave new experiences?". Participants were instructed to rate how accurately the statement reflected how they behaved today on a scale from 0 ("None of the time") to 10 ("All of the time") in increments of 0.1 . The sample-mean of sensation-seeking was $3.12(S D=2.55, \min =0, \max =10)$.

Day's Alcohol Consumption. Alcohol consumption for the previous day was measured during each daily diary assessment using three items of the form, "Yesterday, how many of the following drinks did you consume?" followed with prompts and definitions of standard servings 
for "beer" (12 fl oz), "wine” (5 fl oz), and "shots of liquor" (1.5 fl oz). Responses were given on a $0,1,2,3,4,5+$ response scale for each beverage category and were summed to obtain the total servings of alcohol consumed the previous day (see Lydon et al., 2016 for previous use of this item to assess daily alcohol use). Participants were asked to report on yesterday's alcohol use in order to ensure that alcohol use that may have occurred following completion of the evening survey was captured. On average, participants consumed 0.68 drinks per day $(S D=0.71)$. On drinking days (27\% of all days and with $80.24 \%$ of participants having at least one drinking day), participants drank an average of 2.46 drinks $(S D=1.74)$.

Day's Risk-Taking. Day's risk-taking was measured using two items: "Yesterday, I took more or less risks than I normally do" and "Yesterday, my behaviors was more or less risky than usual". Participants provided their responses on a sliding scale from -50 ("less than usual") to 50 ("more than usual") in increments of 1. Participants were instructed during the laboratory session that a score of -50 did not necessarily mean no risks, it meant much less risky behavior or much less frequent risk-taking than usual for them, and that a score of 0 would indicate a day of the same amount of risky behavior that is typical for them. Intraclass correlation analyses indicated that the proportion of variance associated with within-person variability in risk-taking was 0.56 . The sample-mean of day's risk-taking was $-8.30(S D=20.85, \min =-50$, $\max =50)$. Participants also reported on their riskiest behavior of the day in an open-ended response to the question “What was the riskiest thing you did Yesterday?". Participants were asked to report on yesterday's riskiest behavior in order to ensure risky behaviors that may have occurred following completion of the evening survey were captured.

Trait Sensation-Seeking and Impulsivity. Trait sensation-seeking and impulsivity was measured using the Brief Sensation-Seeking Scale (BSSS; Hoyle et al., 2002) and the Urgency, 
Premeditation, Perseverance, and Sensation-Seeking Scale (UPPS; Whiteside \& Lynam, 2001) during the baseline survey. The BSSS is an 8-item scale and measures sensation-seeking as comprising four components: thrill and adventure seeking, experience seeking, disinhibition, and boredom susceptibility. Items range from 1 ("Disagree Strongly”) to 5 (“Strongly Agree). The mean of all 8 items was calculated and had a sample mean of $3.21(S D=0.80)$. Internal reliability of the BSSS was excellent $(\alpha=0.81)$.

The UPPS is a 45-item scale made up of four subscales: premeditation, urgency, sensation-seeking, and perseverance. Items range from 1 ("Agree Strongly") to 4 ("Disagree Strongly") and were reverse coded, when appropriate, such that higher values on an item indicated higher impulsivity. The mean of each subscale was calculated. Participants reported a mean premeditation of $1.87(S D=0.44)$, a mean urgency of $2.24(S D=0.57)$, a mean sensationseeking of $2.73(S D=0.61)$, and a mean perseverance of $1.80(S D=0.44)$. Internal reliability of the subscales was excellent with $\alpha=0.84,0.88,0.87,0.80$ for premeditation, urgency, sensationseeking, and perseverance, respectively.

\section{Data Analysis}

Data analysis was conducted in four steps. First, we examined the reliability and validity of the sensation-seeking scale used as part of the daily diary. Second, we estimated two separate multilevel models to examine the association between sensation-seeking and alcohol use and risk-taking, respectively. Third, we analyzed daily reports of risky behaviors to identify the types of risks that participants took during the course of daily life. Fourth, we examined associations between trait sensation-seeking and behavior during the daily diary protocol.

Reliability and validity of day's sensation-seeking scale. Two items were available for the day's sensation-seeking scale, allowing us to compute a reliable change score $\left(R_{c}\right)$ designed 
for intensive longitudinal measures (Bolger \& Laurenceau, 2013). $R_{c}$ values may range from 0 to 1 , with values of 0.70 or above representing a scale with high reliability in terms of capturing within-person change over time. After assessing the reliability of the scale, we then computed an intraclass correlation (ICC) to identify the proportion of between-person and within-person variance in the day's sensation-seeking scale. Larger ICC values indicate a higher proportion of total variance at the between-person level.

Although the field lacks a gold standard for assessing the validity of daily scales, the convergent validity of the day's sensation-seeking measure can be examined by comparing the average day's sensation-seeking score across the 21 days with existing trait measures of sensation-seeking. No guidelines exist for determining what correlation would indicate satisfactory validity. Based on the development of a previously published momentary scale of a related construct (impulsivity; Tomko et al., 2014), we chose a threshold of $r=0.30$ as a moderate correlation (Cohen, 1988) indicative of satisfactory validity. Further, we estimated the correlation between the average day's sensation-seeking scale and three impulsivity subscales to provide evidence for discriminant validity.

Associations between sensation-seeking and alcohol use. We tested the extent to which sensation-seeking was associated with alcohol use by implementing a multilevel model. A multilevel model framework (Snijders \& Bosker, 2012) was adopted to accommodate the nested nature of the intensive repeated measures data (21 days nested within 167 persons). In order to allow an examination of both within-person and between-person associations between sensationseeking and alcohol use, sensation-seeking was parameterized to separate within-person and between-person associations by creating time-invariant (between-person) and time-varying (within-person) versions of the sensation-seeking variable (see Bolger \& Laurenceau, 2013). We 
calculated the time-invariant, between-person variable for usual sensation-seeking as the grandmean centered individual mean score of sensation-seeking across all days in the daily diary study. Participants with positive values of usual sensation-seeking had higher than usual levels of sensation-seeking throughout the study compared with other participants in the sample. Participants with negative values of usual sensation-seeking had lower levels of sensationseeking compared with other participants in the sample. We calculated time-varying, day's sensation-seeking variable as deviations from these between-person means. Thus, zero on day's sensation-seeking indicated a day of usual levels of sensation-seeking, negative values indicated days of less than usual levels of sensation-seeking, and positive values indicated days of more than usual levels of sensation-seeking for each individual. The alcohol use variable was slid forward by one day (as the question was phrased to measure previous day's alcohol use to ensure the capture of alcohol use that occurred after the survey) such that day's sensation-seeking represented sensation-seeking on a concurrent day to the reports of alcohol use.

After data preparation, we fit a multilevel hurdle model (Atkins et al., 2013) using glmmTMB in R, specifying a truncated Poisson function (Brooks et al., 2017). Count data such as alcohol use data (i.e., number of drinks consumed) are often positively skewed and include many observations at zero. Indeed, this is the case with the current data (Figure S1). These data violate the distributional assumptions of linear mixed models. Hurdle models include a logistic regression to model the zeroes in the data as well as a count regression (in this case Poisson) to model the counts. All the zeroes (non-alcohol use days) are modeled with the logistic regression and non-zero-counts (alcohol use days) are modeled by a truncated Poisson (i.e., truncated as it does not contain zero). We regressed alcohol use on day's sensation-seeking, usual sensationseeking, and weekend (dummy coded such that Friday, Saturday, and Sunday were indicated by 
1) and specified random intercepts and random slopes for day's sensation-seeking at both the zero and count levels of the model.

Associations between sensation-seeking and risk-taking. We tested the extent to which sensation-seeking was associated with risk-taking using a linear multilevel model. The risktaking variable was slid forward by one day (as the question was phrased to measure previous day's risk-taking to ensure the capture of risk-taking that occurred after the survey) such that day's sensation-seeking represented sensation-seeking on a concurrent day to the reports of risktaking. The risk-taking variable was designed to provide insight into within-person deviations but not between-person information (i.e., the scale was centered at 0 for a day of usual risktaking by design). As such, we included time-varying predictors only. We regressed risk-taking on day's sensation-seeking and weekend. The model was estimated using nlme in R (Pinheiro et al., 2018).

Risk-taking in daily life. To provide insight into the types of risks participants were taking during the course of their daily lives, we used natural language processing methods coupled with network analysis to provide a meaningful description of the vast amount of risktaking entries $(n=3141)$ collected during the daily diary (see also Ji et al., 2015 for an example of the use of this method as a data reduction technique). Days on which no risks were reported (e.g., "I didn't do anything that I would consider risky") were removed, leaving 2490 entries. We tokenized the text of each entry using tidytext (Silge \& Robinson, 2016), segmenting the text of each entry into separate words. We removed stop words that are commonly-used in the English language (e.g., "and", "the", "of") and that were not of interest for quantifying the similarity among entries using a list of words provided through tidytext based on three lexicons of "onix", "SMART", and "snowball". We then removed non-alphabetical characters from the text. We 
used quanteda (Benoit et al., 2017) to reduce words to their word stem form. Taking all entries, we computed term frequency-inverse document frequency (tf-idf) values for each term within each document using text2vec (Selivanov \& Wang, 2018). After calculating the tf-idf for each entry, we quantified the similarity between all pairs of text entries by computing the cosine similarity using the qlcMatric package (Cysouw, 2016). The cosine similarity is a quantification of entry similarity ranging from 0 to 1 , with higher values indicating greater similarity.

We then performed community detection in MATLAB using the brain connectivity toolbox (Rubinov \& Sporns, 2010) on the $2490 \times 2490$ undirected adjacency matrix of the cosine similarity between all possible pairs of risk entries. The aim of the community detection was to assign each text entry to a community within which the nature of the risks described was similar. The algorithm, a Louvain-like locally greedy method, has been demonstrated to provide high quality results (Blondel et al., 2008). The algorithm was applied with a default structural resolution parameter of 1 . As the heuristic is non-deterministic, the algorithm was iterated 100 times. The procedure resulted in 100 vectors indicating the community assignment of each of the 2490 reported risk behaviors for each of the 100 algorithm iterations. Across these 100 vectors, we chose the representative partition of the risk behaviors into communities in a two-step process. First, we calculated the similarity between partitions obtained from the different algorithm iterations using the $z$-score of the Rand coefficient (Traud et al., 2011). For each pair of partitions $\alpha$ and $\beta$, we calculated the Rand z-score in terms of the number of pairs of nodes in the network $M$, the number of pairs $M_{\alpha}$ that are in the same community in partition $\alpha$, the number of pairs $M_{\beta}$ that are in the same community in partition $\beta$, and the number of pairs of nodes $W_{\alpha \beta}$ that have the same community assignment in partition $\alpha$ and partition $\beta$ : 


$$
z_{\alpha \beta}=\frac{1}{\sigma_{W_{\alpha \beta}}} W_{\alpha \beta}-\frac{M_{\alpha} M_{\beta}}{M}
$$

where $\sigma_{W_{\alpha \beta}}$ is the standard deviation of $W_{\alpha \beta}$ (see Doron et al., 2012). We then determined the partition that was the most similar to all other partitions by identifying the partition with the largest average $z$-score and took that forward for the remainder of the analyses. Once text entries had been assigned to communities, we calculated the most frequent words within communities to identify the most common risks captured by each community, and we gave titles to each community that captured the prototypical risks reported in each community. This procedure resulted in 13 communities reflecting domains of risk as well as 7 additional idiosyncratic risks that were not assigned to any community. We make use of the community assignments in the creation of a risk-taking diversity score below.

Sensation-seeking and risk-taking diversity. To examine the association between sensation-seeking and the diversity of risk behaviors reported during the course of daily life, we created a risk-taking diversity index. For each individual, we computed the frequency with which their self-reported riskiest behavior of the day fell into each risk domain identified in the community detection step above (see Risk-taking in daily life section). We then calculated Shannon's diversity index:

$$
\text { RiskTaking Diversity }{ }_{i}=H_{i}=-\sum_{j=1}^{m} p_{i j} \ln p_{i j}
$$

where $m$ is the number of risk communities and $p_{i j}$ is the proportion of participant $i$ 's risk behaviors that were of each discrete risk type, $j=1$ to $m$. Scores can range from 0 to $\ln (m)$, with higher scores indicating greater risk-taking diversity. Then, we ran regression analyses in which this risk-taking diversity index was the dependent variable and was predicted by trait sensation- 
seeking, while controlling for both age and gender and the number of daily diary days completed.

\section{RESULTS}

To examine daily fluctuations in sensation-seeking, impulsivity, and their associations with alcohol use and risk-taking, we use 21-day daily diary data. Out of a possible total of 3507 daily diary days (21 days $\times 167$ participants), $3141(89.56 \%)$ were available. The number of daily diary days completed by participant ranged from 11 to $21(M=18.81, S D=2.75)$. We provide descriptive statistics and zero-order correlations of the variables used in the analyses in Table 1.

[TABLE 1 ABOUT HERE]

\section{Reliability and validity of day's sensation-seeking scale}

The sensation-seeking scale exhibits reliable within-person change $\left(R_{c}=0.85\right)$. Intraclass correlation analyses indicate that a substantial proportion (0.49) of day's sensation-seeking variance is associated with within-person variability in sensation-seeking. The mean of the day's sensation-seeking score shows positive and moderate correlations with two trait measures of sensation-seeking, the Brief Sensation-Seeking Scale $(r=0.40, p<0.001)$ and the UPPS sensationseeking subscale $(r=0.34, p<0.001)$, providing evidence for the scale's convergent validity. Notably, correlations between the day's sensation-seeking scale and the premeditation, perseveration, and urgency subscales of the UPPS are not significant (all $p$-values $>0.05$; Table 1), providing evidence for discriminant validity of the scale. 


\section{Day's sensation-seeking is positively associated with day's alcohol use}

We ran a multilevel hurdle model to examine whether day's sensation-seeking was associated with day's alcohol use. The zero-inflation submodel of the hurdle model estimates the probability of an extra zero (no alcohol use) such that a positive contrast indicates a higher chance of no alcohol use. Days of higher than usual sensation-seeking are more likely to be days on which alcohol is used $(b=-0.09, p=0.02)$ and weekend days are more likely to be alcohol use days relative to weekdays $(b=-0.74, p<0.001)$. There is no association between day in the study and the probability of no alcohol use days $(b=-0.01, p=0.35)$. At the between-person level, there is no association between average sensation-seeking across days in the study and the probability of more or less alcohol use days $(b=-0.04, p=0.57)$.

The conditional submodel of the hurdle model estimates the positive count process, providing insight into variables that increase or decrease the likelihood of consuming more alcohol on alcohol use days. Day's sensation-seeking is not associated with amount of alcohol consumed $(b=0.03, p=0.33)$. Greater amounts of alcohol are consumed on weekend drinking days relative to weekday drinking days $(b=0.32, p<0.001)$. Day of study was not associated with alcohol consumption $(b=0.01, p=0.22)$. At the between-person level, participants with high levels of sensation-seeking across days consumed more alcohol on alcohol use days relative to participants with lower levels of sensation-seeking $(\mathrm{b}=0.09, p=0.02)$.

[TABLE 2 ABOUT HERE]

\section{Day's sensation-seeking is positively associated with day's risk-taking}


The risk-taking scale exhibited reliable within-person change $R_{c}=0.88$. We ran multilevel models to examine whether day's sensation-seeking was associated with day's risk-taking (Table 2). Days of higher than usual sensation-seeking are also days of higher than usual risk-taking $(\mathrm{b}=0.64, p=0.01)$. Risk-taking is higher than usual on weekends relative to weekdays $(\mathrm{b}=1.44$, $p=0.01)$ and increases over the course of the study $(\mathrm{b}=0.14, p=0.01)$.

\section{[TABLE 3 ABOUT HERE]}

\section{Risks in daily life}

Participants report riskier behavior on days of higher than usual sensation-seeking $(b=0.64, p=0.01$; Table 2$)$. To provide insight on the types of risks that participants engaged in during the daily diary protocol, we created a visualization of the network resulting from the cosine similarity analysis on the self-reported riskiest behaviors of the day (Figure 1). Nodes represent individual reports and edges represent the cosine similarity between reports. Twenty communities were identified by community detection. Seven communities contained only one risky behavior; these were highly idiosyncratic risk behaviors that occurred only once and included "dissected fetal pig" and "tanned". The community allegiance of the nodes of the other thirteen communities is indicated by color. A list of the top five most frequent words associated with the self-reports within each community is shown. Below we name the communities and describe prototypical behaviors associated with each community.

The largest community, comprising $13.73 \%$ of risk reports, largely contains schoolrelated risks and includes reports such as "Procrastinated on studying for exam by watching Netflix", "Put off studying", and "accepted my study abroad". The second largest community comprises $11.85 \%$ of risks and contains a large proportion of reports associated with engagement 
in novel experiences and some associated with overindulgence, in particular those related to food. Reports include "trying new dance moves", "I tried a new recipe", "checked out a new city i [sic] might move to", "watched a new tv show", "took on a new volunteer opportunity", and "Ate ice cream even though I'm probably lactose intolerant". The third largest community comprising $11.61 \%$ of risk reports contains many reports of social risks such as: "speak to someone I feared”, “I told someone I didn't know very well about personal information”, “Trusted someone who was untrustworthy in the past", "Told someone on the bus to shut up", “told my boss I wouldn't be happy if he hired someone else", "decided to break things off with someone I've been dating", and "I gave my number to a boy I met at the Penn relays". Work risks largely make up the next community comprising $11.45 \%$ of risk reports. Reports include: "I intentionally didn't finish work I needed to, knowing I would miss a soft deadline", "left work frequently for errands", "took on a difficult work project", "Try a different style of working at my job", and "left work early". The next largest community contains exploratory risks (11.33\%) and consists of idiosyncratic forms of exploration: "went out drinking with strangers", "I hiked in the woods", "went on a whale watching tour", "went to a new place", and "went to a party where I knew only a few people”. The next largest community $(9.88 \%)$ consists of walking risks on the theme of walking in the city, often at night: "walked home in the dark", "walked across campus in a crazy nor-easter [sic]", "Walked ten blocks during a hailstorm", "walked in an unsafe are [sic] of town", and "went for a walk despite injured leg”. The next largest community $(9.12 \%)$ consists mainly of transportation risks, in particular risky driving and biking: "Ride my bike the wrong way on a one way street to get to work faster", "Rode a bike without a helmet", "drove while not paying attention", "Drove very fast in order to get to an appointment that I was late for", "I drove without a seatbelt", and "I drove recklessly". The next community (7.27\%), 
friend risks, consists of behaviors involving experiences with friends and includes reports such as: "went last minute to meet friends for a drink", "emotionally risky... made myself vulnerable with a friend", "I lied to a friend and cancelled plans with her", and "tell a friend she was wrong”. This community had similar social content to the social risks community but with greater emphasis on friends specifically. A responsibilities community (5.94\%) encompasses instances in which responsibilities (in particular related to school) were not met: "didn't go to a meeting I knew would be boring", "Did not go into my lab when I was supposed to", and "didn't do class readings". Alcohol risks make up the majority of the next community $(2.93 \%)$ with reports such as: “drank too much”, "Drank champagne while sick”, “drank more wine than I typically do", and "drank a beer outside on the street". The next largest community entails driving risks (2.53\%) and is highly similar to the transportation risks community, although with an almost exclusive focus on driving cars: "drive aggressively through a parade", "almost missed a stop sign when driving and had to slam my breaks [sic]", and "Glanced at my phone while driving". A jaywalking risks community (1.37\%) exclusively contains descriptions of jaywalking, often simply the word "jaywalk". Finally, the last community $(0.72 \%)$ is comprised of smoking risks and contains reports such as: "smoked weed", "smoke hookah", and "smoke".

\section{[FIGURE 1 ABOUT HERE]}

\section{Trait sensation-seeking and diversity of risk behaviors}

Using the thirteen communities of risk (Figure 1), as well as an additional "Other risk" category in which the 7 risk communities consisting of only one risk were combined, we calculated Shannon's diversity index as a measure of risk-taking diversity. Scores on both the 
BSSS and the sensation-seeking subscale of the UPPS positively correlate with this risk-taking diversity score $(r=0.26, p<0.001$ and $r=0.18, p=0.02$, respectively). Both the BSSS and the sensation-seeking subscale of the UPPS remain significantly associated with the risk-taking diversity score in regression analyses controlling for age, gender, and the number of days of the daily diary protocol completed (Table 4).

\section{[TABLE 4 ABOUT HERE]}

\section{Robustness and additional analyses}

Additional analyses confirm that results for alcohol use are robust to the inclusion of participants exhibiting no alcohol use throughout the 21 days and who thus could not provide insight into the within-person association between sensation-seeking and alcohol use (see LydonStaley et al., 2017; Ram et al., 2017; Table S1). Results for alcohol use and risk-taking are also robust to the inclusion of previous day's sensation-seeking as well as controlling for previous day's alcohol use (Table S2) and previous day's risk-taking (Table S3). We also examined the extent to which the association between day's sensation-seeking and day's risk-taking may be mediated through day's alcohol use and find evidence for a significant indirect effect (Table S4). Approximately $19 \%$ of the association between day's sensation-seeking and day's risk-taking is mediated through alcohol, such that days of higher than usual sensation-seeking are also days of higher than usual alcohol use which are, in turn, days of higher than usual risk-taking (Figure S2). 


\section{Discussion}

Sensation-seeking is associated with engagement in risky behaviors (Hittner \& Swickert, 2006; Lydon-Staley \& Geier, 2018) and exhibits marked intraindividual change over development (Lynne-Landsman et al., 2011; Steinberg et al., 2018). Despite theorized roles for short-term, within-person fluctuations in sensation-seeking in risk-taking (Lydon-Staley \& Bassett, 2018; Neary, 1975; Zuckerman, 1994) and the necessity of considering both intraindividual change and intraindividual variability in order to thoroughly characterize individuals (Nesselroade, 1991), little work has considered intraindividual variability in sensation-seeking. We measured naturalistic day-to-day fluctuations in sensation-seeking over the course of 21 days and tested within-person associations between sensation-seeking and both alcohol use and self-defined risky behavior. Our measure of sensation-seeking was capable of reliably measuring within-person change $\left(R_{c}=0.85\right)$ and exhibited substantial within-person variance, with approximately $46 \%$ of the variance in sensation-seeking across 21 days associated with within-person, as opposed to between-person, variance. In addition, the scale exhibited convergent validity in the form of moderate, positive correlations with two trait sensationseeking scales. Notably, the magnitude of these correlations is in line with estimates of convergent validity between momentary and trait scales for related constructs (impulsivity) with double the number of momentary items (Tomko et al., 2014). In addition, evidence for discriminant validity emerged such that the average day's sensation-seeking score was not correlated with impulsivity subscales of the UPPS. Thus, the measure of day's sensation-seeking is short, reliably captures within-person fluctuations, exhibits satisfactory convergent validity with two trait sensation-seeking scales, and exhibits satisfactory divergent validity with measures of trait impulsivity. 
Capturing fluctuations in sensation-seeking allowed us to disaggregate within-person and between-person variance in sensation-seeking and to test within-person theories of the role of sensation-seeking in risk behaviors (Lydon-Staley \& Bassett, 2018). Consistent with the hypothesized importance of state sensation-seeking in promoting risky behavior, days of higher than usual sensation-seeking were also more likely to be days of alcohol use relative to days of no alcohol use. Day's sensation-seeking was not associated with the amount of alcohol consumed on drinking days. Instead, there was an association at the between-person level, such that participants high in sensation-seeking were more likely to consume more alcohol on drinking days relative to participants lower in sensation-seeking. The distinct associations between sensation-seeking and alcohol use at the between-person and within-person levels highlight the importance of disentangling within-person and between-person variance in order to make within-person inferences, as findings observed at the between-person level cannot be assumed to generalize to the within-person level (Hamaker, 2012).

Also consistent with our hypotheses was the finding that days of higher than usual sensation-seeking were also days of higher than usual engagement in self-defined risky behavior. By having participants report on the riskiest behavior they engaged in each day, we additionally provide novel insight into the risks that individuals undertake during the course of daily life. Only a small portion (2.93\%) of self-reported risks concerned alcohol use risks. Other substance use, in particular smoking, also emerged as a risk domain and, similar to alcohol use, concerned a small portion $(0.72 \%)$ of reported risks. These results highlight that a focus on substance use when considering risk behavior fails to capture the majority of behaviors participants perceive to be risky in their daily lives and illustrates the importance of considering the subjective nature of risk (Hansson et al., 2010; Redmill, 2002) alongside investigator-defined constructs of risk. A 
large portion of risk-taking entailed engagement in novel experiences (11.85\%), social risks (11.61\%), and exploratory behaviors (11.33\%), highlighting domains of risk that warrant greater attention in future research. The broad array of risks was associated with potential losses ("decided to walk home rather than take an Uber at the risk of being late to a dinner", "walked by myself at $11 \mathrm{pm}$ in an unsafe neighborhood while carrying my laptop in my bookbag”). Risktaking also resulted in potential benefits to the participants and those around them ("called out discrimination", “Asked a stranger if he was okay. He was crying”, "I tried pureed watermelon on my oatmeal for the first time... it was really good!", "I brought together two groups of friends and it worked out"). The many positive risks highlight the role within-person increases in sensation seeking plays in motivating positive risk-taking (i.e., socially acceptable) and, in the process, the enrichment of lives with new and enjoyable experiences (Duell \& Steinberg, 2018). Although the main focus of the study was on the within-person associations between sensation-seeking and both alcohol use and risk-taking, we collected data on trait sensationseeking. This experimental design allowed us to examine how trait sensation-seeking was associated with behavior during the course of daily life. As hypothesised, participants high in trait sensation-seeking reported greater diversity in the categories of risk behaviors that they engaged in during the daily diary period. This diversity of risk engagement is in line with the emphasis on the tendency to seek out novel experiences at the core of conceptualizations of trait sensation-seeking. These findings add to perspectives that consider the positive side of sensationseeking (Hansen \& Breivik, 2001; Yoneda et al., 2019) whereby the increased tendency of sensation-seekers to take risks in order to experience novel and exciting experiences leads to potentially detrimental outcomes (e.g., addiction) but also potential benefits (e.g., the accrual of new skills). 


\section{Limitations and future outlook}

It is important to consider the findings in light of the study's strengths and limitations. Our use of daily diaries allowed us to capture naturally-occurring fluctuations in sensationseeking, alcohol use, and risky behavior during life as it is lived (Bolger et al., 2003). However, the daily diary data are limited in their ability to evaluate temporal precedence. Future work, drawing on multiple occasions ( 3 or more) each day, will provide greater possibilities to examine putative causal associations. Our measure of sensation-seeking is based on two items and, thus, may not capture the full breadth of the construct. However, it is capable of reliably capturing within-person change and shows satisfactory convergent and discriminant validity with two trait sensation-seeking scales while being short enough (2-items) to be readily accommodated in experience-sampling designs.

\section{Conclusions}

In summary, we extend previous examinations of the association among sensationseeking and risk behaviors by measuring naturalistic fluctuations in sensation-seeking during the course of daily life and demonstrating that days of higher than usual sensation-seeking are also more likely to be days of alcohol use than no alcohol use and also days of riskier behavior than usual. We also provide a detailed examination of the types of risks that are taken during everyday life. The findings highlight the importance of considering short-term dynamics in sensation-seeking and the subjective nature of risk in order to understand risk behaviors during the course of daily life. 


\section{References}

Ashenhurst, J. R., Harden, K. P., Corbin, W. R., \& Fromme, K. (2015). Trajectories of binge drinking and personality change across emerging adulthood. Psychology of Addictive Behaviors, 29, 978-991.

Atkins, D. C., Baldwin, S. A., Zheng, C., Gallop, R. J., \& Neighbors, C. (2013). A tutorial on count regression and zero-altered count models for longitudinal substance use data. Psychology of Addictive Behaviors, 27, 166-177.

Benoit, K., Watanabe, K., Nulty, P., Obeng, A., Wang, H., Lauderdale, B., \& Lowe, W. (2017). quanteda: Quantitative analysis of textual data. R package version: 0.9.9-65. http://quanteda.io

Blondel, V. D., Guillaume, J. L., Lambiotte, R., \& Lefebvre, E. (2008). Fast unfolding of communities in large networks. Journal of Statistical Mechanics: Theory and Experiment, 2008(10), P10008.

Bolger N, Davis A, Rafaeli E (2003). Diary methods: Capturing life as it is lived. Annual Review of Psychology, 54, 579-616.

Bolger, N., \& Laurenceau, J. P. (2013). Intensive longitudinal methods. New York, NY: Guilford.

Brooks, M. E., Kristensen, K., van Benthem, K. J., Magnusson, A., Berg, C. W., Nielsen, A., ... , \& Bolker, B. M. (2017). glmmTMB balances speed and flexibility among packages for zero-inflated generalized linear mixed modeling. The R journal, 9, 378-400.

Carver, C. S., \& White, T. L. (1994). Behavioral inhibition, behavioral activation, and affective responses to impending reward and punishment: the BIS/BAS scales. Journal of Personality and Social Psychology, 67, 319-333. 
Cohen, J. (1988). Statistical Power Analysis for the Behaviorsl Sciences. New York, NY:

Routledge Academic.

Coskunpinar, A., Dir, A. L., \& Cyders, M. A. (2013). Multidimensionality in impulsivity and alcohol use: A meta-analysis using the UPPS model of impulsivity. Alcoholism: Clinical and Experimental Research, 37, 1441-1450.

Costa, P. T., Jr., \& McCrae, R. R. (1992). Revised NEO personality inventory (NEO PI-R) and NEO five-factor inventory (NEO-FFI) professional manual. Odessa, FL: Psychological Assessment Resources, Inc.

Curran, P. J., \& Bauer, D. J. (2011). The disaggregation of within-person and between-person effects in longitudinal models of change. Annual Review of Psychology, 62, 583-619.

Cyders, M. A., Flory, K., Rainer, S., \& Smith, G. T. (2009). The role of personality dispositions to risky behavior in predicting first-year college drinking. Addiction, 104, 193-202.

Cysouw, M. (2015). qlcMatrix: Utility sparse matrix functions for quantitative language comparison. $\mathrm{R}$ package version 0.9 .5 . https:/CRAN.R-project.org/package=qlcMatrix

Csikszentmihalyi, M., Larson, R., \& Prescott, S. (1977). The ecology of adolescent activity and experience. Journal of Youth and Adolescence, 6, 281-294.

Donohew, L., Zimmerman, R., Cupp, P. S., Novak, S., Colon, S., \& Abell, R. (2000). Sensation seeking, impulsive decision-making, and risky sex: Implications for risk-taking and design of interventions. Personality and Individual Differences, 28, 1079-1091.

Doron, K. W., Bassett, D. S., \& Gazzaniga, M. S. (2012). Dynamic network structure of interhemispheric coordination. Proceedings of the National Academy of Sciences, 109, 18661-18668. 
Duell, N., \& Steinberg, L. (2019). Positive risk taking in adolescence. Child Development Perspectives, 13(1), 48-52.

Fisher, C. D., \& To, M. L. (2012). Using experience sampling methodology in organizational behavior. Journal of Organizational Behavior, 33(7), 865-877.

Hamaker, E. L. (2012). Why researchers should think "within-person": A paradigmatic rationale. In M.R. Mehl \& T.S. Conner (Eds.), Handbook of research methods for studying daily life (pp. 43-61). New York, NY: Guilford.

Hansen, E. B., \& Breivik, G. (2001). Sensation seeking as a predictor of positive and negative risk behaviour among adolescents. Personality and Individual Differences, 30(4), 627640.

Hansson, S. O. (2010). Risk: objective or subjective, facts or values. Journal of Risk Research, $13,231-238$.

Hittner, J. B., \& Swickert, R. (2006). Sensation seeking and alcohol use: A meta-analytic review. Addictive Behaviors, 31, 1383-1401.

Hoyle, R. H., Stephenson, M. T., Palmgreen, P., Pugzles Lorch, E., \& Donohew, R. L. (2002). Reliability and validity of a brief measure of sensation seeking. Personality and Individual Differences, 32, 401-414.

Jack, S. J., \& Ronan, K. R. (1998). Sensation seeking among high-and low-risk sports participants. Personality and Individual Differences, 25, 1063-1083.

Jacomy, M., Venturini, T., Heymann, S., \& Bastian, M. (2014). ForceAtlas2, a continuous graph layout algorithm for handy network visualization designed for the Gephi software. PloS one, 9, e98679. 
Ji, X., Machiraju, R., Ritter, A., \& Yen, P. Y. (2015). Examining the Distribution, Modularity, and Community Structure in Article Networks for Systematic Reviews. In AMIA Annual Symposium Proceedings (Vol. 2015, p. 1927). American Medical Informatics Association.

Larson, R., \& Csikszentmihalyi, M. (2014). The experience sampling method. In Flow and the foundations of positive psychology (pp. 21-34). Dordrecht, NL: Springer.

Lydon-Staley, D. M., Zurn, P., \& Bassett, D. S. (2018). Inconsistent curiosity: Augmentation and blunting of curiosity in daily life and implications for well-being. PsyArxiv, https://doi.org/10.31234/osf.io/2vf94

Lydon-Staley, D.M., \& Bassett, D.S. (2018). The promise and challenges of intensive longitudinal designs for imbalance models of adolescent substance use. Frontiers in Psychology, 9 .

Lydon-Staley, D. M., \& Geier, C. F. (2018). Age-Varying Associations Between Cigarette Smoking, Sensation Seeking, and Impulse Control Through Adolescence and Young Adulthood. Journal of Research on Adolescence, 28, 354-367.

Lydon-Staley, D. M., Ram, N., Brose, A., \& Schmiedek, F. (2017). Reduced impact of alcohol use on next-day tiredness in older relative to younger adults: A role for sleep duration. Psychology and Aging, 32, 642-653.

Lydon, D. M., Ram, N., Conroy, D. E., Pincus, A. L., Geier, C. F., \& Maggs, J. L. (2016). The within-person association between alcohol use and sleep duration and quality in situ: An experience sampling study. Addictive Behaviors, 61, 68-73. 
Lynne-Landsman, S. D., Graber, J. A., Nichols, T. R., \& Botvin, G. J. (2011). Is sensation seeking a stable trait or does it change over time? Journal of Youth and Adolescence, 40, 48-58.

Miller, J., Flory, K., Lynam, D., \& Leukefeld, C. (2003). A test of the four-factor model of impulsivity-related traits. Personality and Individual Differences, 34, 1403-1418.

Molenaar, P. C. (2004). A manifesto on psychology as idiographic science: Bringing the person back into scientific psychology, this time forever. Measurement, 2, 201-218.

Neary, R.S. (1975). The development and validation of a state measure of sensation-seeking. Unpublished doctoral dissertation, University of Delaware.

Nesselroade, J.R. (1991). The warp and woof of the developmental fabric. In R. Downs, L. Liben, \& D. Palermo (Eds.), Visions of development, the environment, and aesthetics: The legacy of Joachim F. Wohlwill (pp. 213-240). Hillsdale, NJ: Erlbaum.

Pew Research Center. (2017). Mobile fact sheet. Pew Research Center: Internet, Science \& Tech.

Pinheiro J, Bates D, DebRoy S, Sarkar D and R Core Team (2018). nlme: Linear and Nonlinear Mixed Effects Models. R package version 3.1-137. Retrieved from: https://CRAN.Rproject.org/package $=$ nlme.

Quinn, P. D., \& Harden, K. P. (2013). Differential changes in impulsivity and sensation seeking and the escalation of substance use from adolescence to early adulthood. Development and Psychopathology, 25, 223-239.

Ram, N., Brinberg, M., Pincus, A. L., \& Conroy, D. E. (2017). The questionable ecological validity of ecological momentary assessment: Considerations for design and analysis. Research in Human Development, 14, 253-270. 
Ram, N., \& Gerstorf, D. (2009). Time-structured and net intraindividual variability: tools for examining the development of dynamic characteristics and processes. Psychology and Aging, 24, 778-791.

Redmill, F. (2002). Risk analysis-a subjective process. Engineering Management Journal, 12, 91-96.

Reyna, V.F., \& Huettel, S.A. (2014). Reward, representation, and impulsivity: A theoretical framework for the neuroscience of risky decision-making. In V.F. Reyna \& V. Zayas (Eds.), The Neuroscience of Risky Decision Making (pp.11-42). Washington, D.C.: American Psychological Association.

Rubinov, M., \& Sporns, O. (2010). Complex network measures of brain connectivity: uses and interpretations. Neuroimage, 52, 1059-1069.

Selivanov, D., \& Wang, Q. (2018). text2vec: Modern text mining framework for R. R package version 0.5.0. https://CRAN.R-project.org/package=text2vec

Schwarz, N. (2007). Retrospective and concurrent self-reports: The rationale for real-time data capture. In A. Stone, S. Shiffman, A. Atienza, \& L. Nebeling (Eds.), The science of realtime data capture: Self-reports in health research (pp. 11-26). New York, NY: Oxford University Press.

Silge, J., \& Robinson, D. (2016). tidytext. Text mining and analysis using tidy data principles in R. The Journal of Open Source Software, 1, 37.

Snijders, T. A. B., \& Bosker, R. J. (2012). Multilevel analysis: An introduction to basic and advanced multilevel modeling (2nd ed.). London, United Kingdom: Sage.

Sperry, S. H., Lynam, D. R., \& Kwapil, T. R. (2018). The convergence and divergence of impulsivity facets in daily life. Journal of Personality, 86, 841-852. 
Steinberg, L., Icenogle, G., Shulman, E. P., Breiner, K., Chein, J., Bacchini, D., ... \& Fanti, K. A. (2018). Around the world, adolescence is a time of heightened sensation seeking and immature self-regulation. Developmental Science, 21, e12532.

Tomko, R. L., Solhan, M. B., Carpenter, R. W., Brown, W. C., Jahng, S., Wood, P. K., \& Trull, T. J. (2014). Measuring impulsivity in daily life: the momentary impulsivity scale. Psychological Assessment, 26, 339-349.

Traud, A. L., Kelsic, E. D., Mucha, P. J., \& Porter, M. A. (2011). Comparing community structure to characteristics in online collegiate social networks. SIAM Review, 53, 526543.

Whiteside, S. P., \& Lynam, D. R. (2001). The five factor model and impulsivity: Using a structural model of personality to understand impulsivity. Personality and Individual Differences, 30, 669-689.

Yoneda, T., Ames, M. E., \& Leadbeater, B. J. (2019). Is there a positive side to sensation seeking? Trajectories of sensation seeking and impulsivity may have unique outcomes in young adulthood. Journal of Adolescence, 73, 42-52.

Zuckerman, M. (1994). Behavioral expressions and biosocial bases of sensation seeking. New York, NY: Cambridge University Press. 
RUNNING HEAD: SENSATION-SEEKING AND RISK

Table 1.

Correlations and Descriptive Statistics of Key Study Variables

\begin{tabular}{|c|c|c|c|c|c|c|c|c|c|}
\hline Variables & 1 & 2 & 3 & 4 & 5 & 6 & 7 & 8 & 9 \\
\hline 1. Sensation-seekinga & - & & & & & & & & \\
\hline 2. Alcohol use & $0.16^{*}$ & - & & & & & & & \\
\hline 3. Risk-takinga & 0.09 & 0.12 & - & & & & & & \\
\hline 4. BSSS & $0.40 * * *$ & $0.26 * * *$ & 0.01 & - & & & & & \\
\hline 5. UPPS-SS & $0.34 * * *$ & 0.12 & -0.13 & $0.72 * * *$ & - & & & & \\
\hline 6. UPPS-PREM & 0.08 & 0.11 & -0.02 & $0.51 * * *$ & $0.36 * * *$ & - & & & \\
\hline 7. UPPS-PERS & -0.03 & 0.02 & 0.02 & 0.14 & -0.01 & $0.37 * * *$ & - & & \\
\hline 8. UPPS-URGE & 0.05 & 0.10 & 0.07 & $0.28 * * *$ & 0.10 & $0.48 * * *$ & $0.48 * * *$ & - & \\
\hline 9. Age & $-0.18 *$ & 0.07 & $-0.45 * * *$ & -0.07 & -0.03 & 0.14 & 0.00 & 0.00 & - \\
\hline Mean & 3.15 & 0.68 & -8.44 & 3.21 & 2.73 & 1.87 & 1.80 & 2.24 & 25.19 \\
\hline Standard deviation & 1.91 & 0.71 & 14.43 & 0.80 & 0.61 & 0.44 & 0.44 & 0.57 & 6.89 \\
\hline
\end{tabular}

Notes: Intraindividual mean of the daily diary time series; $N=167 . * * * p<0.001 ;{ }^{*} p<0.01 ; * p<0.05$. BSSS = brief sensation-seeking scale; UPPS = Urgency, Premeditation, Perseverance, and Sensation-seeking Scale; SS = Sensation-seeking; PREM = premeditation; PERS $=$ perseverance; URGE $=$ urgency . 
RUNNING HEAD: SENSATION-SEEKING AND RISK

Table 2

Results of the multilevel hurdle model examining associations with alcohol use

\begin{tabular}{|c|c|c|c|}
\hline \multicolumn{4}{|c|}{ CONDITIONAL SUBMODEL } \\
\hline & Estimate & Standard Error & p-value \\
\hline Intercept & $0.29 * * *$ & 0.08 & $<0.001$ \\
\hline Day's sensation seeking & 0.03 & 0.03 & 0.33 \\
\hline Weekend & $0.32 * * *$ & 0.07 & $<0.001$ \\
\hline Day of study & 0.01 & 0.01 & 0.22 \\
\hline Usual sensation seeking & $0.09 *$ & 0.04 & 0.02 \\
\hline \multicolumn{4}{|c|}{ ZERO-INFLATION SUBMODEL } \\
\hline & Estimate & Standard Error & p-value \\
\hline Intercept & $1.90 * * *$ & 0.16 & $<0.001$ \\
\hline Day's sensation seeking & $-0.09 *$ & 0.04 & 0.02 \\
\hline Weekend & $-0.74 * * *$ & 0.11 & $<0.001$ \\
\hline Day of study & -0.01 & 0.01 & 0.35 \\
\hline Usual sensation seeking & -0.04 & 0.08 & 0.57 \\
\hline \multicolumn{4}{|l|}{ FIT INDICES } \\
\hline \multirow{2}{*}{$\begin{array}{l}\text { AIC } \\
\text { BIC }\end{array}$} & 4819.30 & & \\
\hline & 4914.00 & & \\
\hline
\end{tabular}

Notes: $N=2737$ days nested within 167 participants. 
Table 3

Results of the multilevel model examining associations with day's self-reported risk-taking

\begin{tabular}{l|lll}
\hline \multicolumn{5}{l}{ FIXED EFFECTS } & Estimate & Standard Error & p-value \\
\hline & $-9.26^{* * *}$ & 1.17 & $<0.001$ \\
Intercept & $0.65^{*}$ & 0.26 & 0.01 \\
Day's sensation-seeking & $1.44^{*}$ & 0.59 & 0.01 \\
Weekend & $0.14^{*}$ & 0.05 & 0.01 \\
Day of study & \multicolumn{3}{l}{} \\
\hline FIT INDICES & 22900.29 & & \\
\hline AIC & 22953.51 & & \\
BIC
\end{tabular}

Notes: $N=2737$ days nested within 167 participants. 
Table 4.

Results of the multiple regression analyses examining associations between the Brief SensationSeeking Scale and risk-taking diversity (top) and the UPPS sensation-sensation-seeking subscale and risk-taking diversity (below).

\begin{tabular}{l|lll}
\hline \multicolumn{4}{c}{ Risk-Taking Diversity and Brief Sensation-Seeking Scale } \\
\hline & Estimate & Standard Error & $p$-value \\
\hline Intercept & $0.29 * * *$ & 0.32 & 0.38 \\
BSSS & $0.17^{* * *}$ & 0.05 & $<0.001$ \\
Age & $-0.02^{* *}$ & 0.01 & 0.001 \\
Gender male & -0.13 & 0.10 & 0.19 \\
Gender other & 0.31 & 0.35 & 038 \\
Number of days & $0.04 * *$ & 0.01 & 0.004 \\
$\boldsymbol{R}^{2}$ & 0.17 & & \\
$\boldsymbol{F}$ & $6.47^{* * *}$ & & \\
\hline \multicolumn{5}{|c|}{ Risk-Taking Diversity and UPPS Sensation-Seeking Subscale } \\
\hline Intercept & Estimate & Standard Error & 0.26 \\
UPPS-SS & 0.38 & 0.34 & 0.006 \\
Age & $0.18^{* *}$ & 0.06 & $<0.001$ \\
Gender male & $-0.02^{* * *}$ & 0.01 & 0.17 \\
Gender other & -0.14 & 0.10 & 0.37 \\
Number of days & 0.32 & 0.36 & 0.01 \\
$\boldsymbol{R}^{2}$ & $0.04 * *$ & 0.01 & \\
$\boldsymbol{F}$ & 0.14 & & \\
\hline
\end{tabular}

Notes: Age was sample-mean centered. Gender was a factor variable with female as the reference category. BSSS = brief sensation-seeking scale; UPPS = Urgency, Premeditation, Perseverance, and Sensation-Seeking Scale; SS = sensation-seeking. $* * * p<0.001, * * p<0.01$, $p<0.05 . N=167$. 
Figure 1.

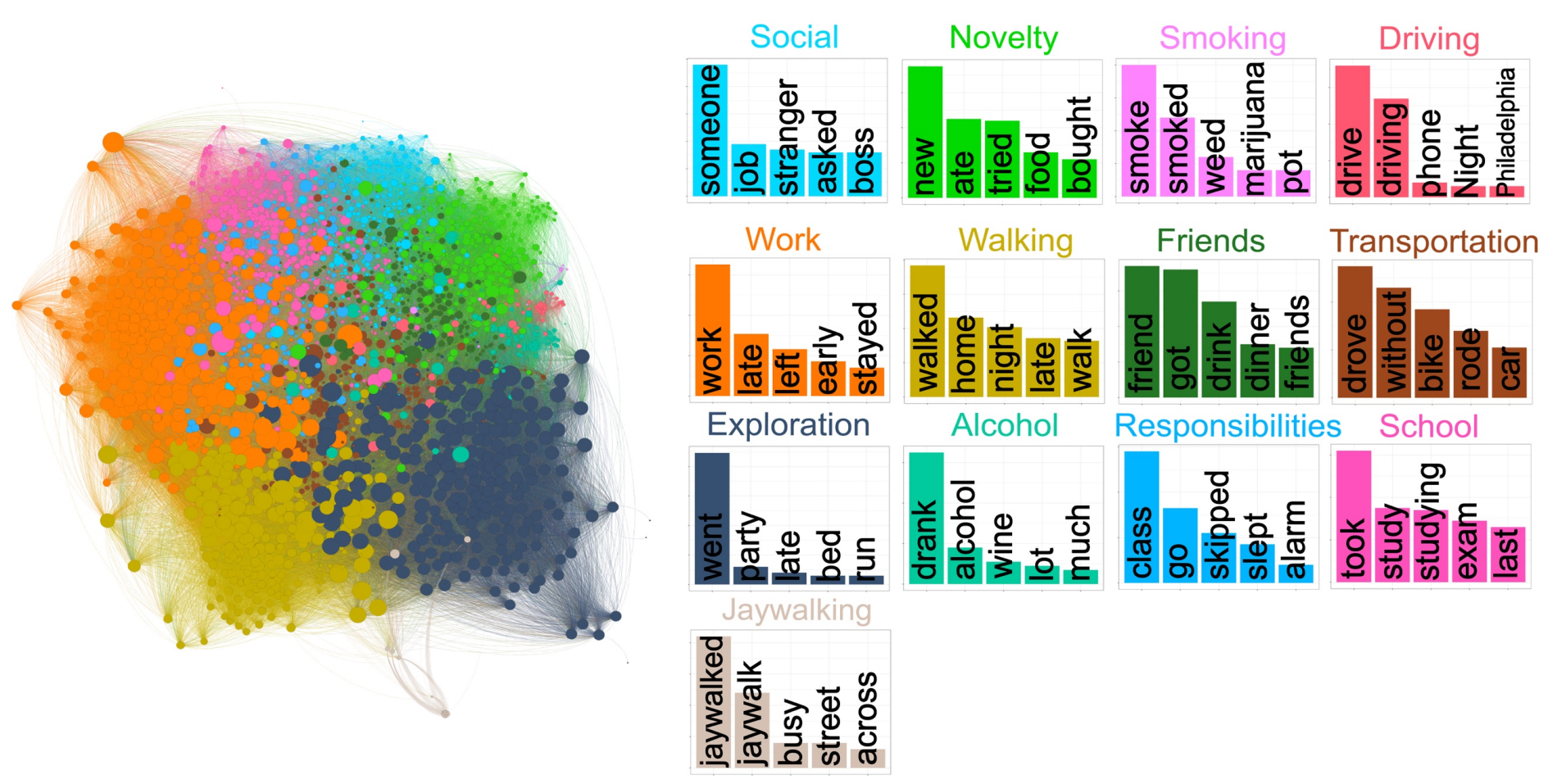

Figure 1. Semantic network of riskiest behaviors of the day. Each node represents one of 2490 self-reports of the riskiest action that participants performed on the previous day. Edges reflect cosine similarity based on term frequency document inverse frequency between reports. Nodes were placed according to a force directed layout (Jacomy et al., 2014) and are colored to illustrate community assignment. The top five most frequent words associated with each community are shown in bar plots. 


\section{Supplementary Material}

Table S1.

Results of the multilevel model examining associations with day's alcohol use with participants reporting no alcohol use throughout the study excluded

\begin{tabular}{|c|c|c|c|}
\hline \multicolumn{4}{|c|}{ CONDITIONAL SUBMODEL } \\
\hline & Estimate & Standard Error & $p$-value \\
\hline Intercept & $0.29 * * *$ & 0.08 & $<0.001$ \\
\hline Day's sensation seeking & 0.03 & 0.03 & 0.33 \\
\hline Weekend & $0.32 * * *$ & 0.07 & $<0.001$ \\
\hline Day of study & 0.01 & 0.01 & 0.22 \\
\hline Usual sensation seeking & $0.09 *$ & 0.04 & 0.02 \\
\hline \multicolumn{4}{|c|}{ ZERO-INFLATION SUBMODEL } \\
\hline & Estimate & Standard Error & $p$-value \\
\hline Intercept & $1.17 * * *$ & 0.12 & $<0.001$ \\
\hline Day's sensation seeking & $-0.08 *$ & 0.04 & 0.02 \\
\hline Weekend & $-0.73 * * *$ & 0.11 & $<0.001$ \\
\hline Day of study & -0.01 & 0.01 & 0.34 \\
\hline Usual sensation seeking & -0.03 & 0.06 & 0.55 \\
\hline \multicolumn{4}{|l|}{ FIT INDICES } \\
\hline AIC & 4661.00 & & \\
\hline BIC & 4751.70 & & \\
\hline
\end{tabular}

Notes: $N=2140$ days nested within 131 participants. 
Table S2.

Results of the multilevel model examining associations with alcohol use controlling for previous day's sensation-seeking and alcohol use

\begin{tabular}{|c|c|c|c|}
\hline \multicolumn{4}{|l|}{ CONDITIONAL SUBMODEL } \\
\hline & Estimate & Standard Error & $p$-value \\
\hline Intercept & $0.23^{*}$ & 0.09 & 0.02 \\
\hline Day's sensation seeking & 0.04 & 0.03 & 0.21 \\
\hline Weekend & $0.33 * * *$ & 0.07 & $<0.001$ \\
\hline Day of study & 0.01 & 0.01 & 0.06 \\
\hline Usual sensation seeking & $0.09 *$ & 0.04 & 0.04 \\
\hline Previous day's sensation seeking & -0.01 & 0.02 & 0.53 \\
\hline Previous day's alcohol use & -0.01 & 0.02 & 0.56 \\
\hline \multicolumn{4}{|c|}{ ZERO-INFLATION SUBMODEL } \\
\hline & Estimate & Standard Error & $p$-value \\
\hline Intercept & $1.95 * * *$ & 0.17 & $<0.001$ \\
\hline Day's sensation seeking & $-0.09 *$ & 0.05 & 0.04 \\
\hline Weekend & $-0.77 * * *$ & 0.12 & $<0.001$ \\
\hline Day of study & -0.01 & 0.01 & 0.30 \\
\hline Usual sensation seeking & -0.02 & 0.08 & 0.76 \\
\hline Previous day's sensation seeking & -0.01 & 0.03 & 0.83 \\
\hline Previous day's alcohol use & -0.03 & 0.04 & 0.43 \\
\hline \multicolumn{4}{|l|}{ FIT INDICES } \\
\hline AIC & 4229.90 & & \\
\hline BIC & 4345.70 & & \\
\hline
\end{tabular}

Notes: $N=2140$ days nested within 131 participants. 
Table 3.

Results of the multilevel model examining associations with day's self-reported risk-taking controlling for previous day's sensation-seeking and risk-taking

\begin{tabular}{l|lll}
\hline \multicolumn{5}{l}{ FIXED EFFECTS } & Estimate & Standard Error & p-value \\
\hline & $-7.26^{* * *}$ & 0.97 & $<0.001$ \\
Intercept & $0.81^{* *}$ & 0.27 & 0.003 \\
Day's sensation seeking & 0.91 & 0.58 & 0.12 \\
Weekend & $0.15^{* *}$ & 0.05 & 0.003 \\
Day of study & $-0.49^{* *}$ & 0.18 & 0.007 \\
Previous day's sensation seeking & $0.22^{* * *}$ & 0.02 & $<0.001$ \\
Previous day's risk-taking & & & \\
\hline FIT INDICES & 20141.83 & & \\
\hline AIC & 20205.46 & & \\
BIC &
\end{tabular}


Table S4.

Mediation model examining the within-person associations among sensation-seeking, impulsivity, and risk-taking

\begin{tabular}{l|ll}
\hline & Estimate & Confidence Interval \\
\hline Sensation seeking $\rightarrow$ alcohol use $\left(\boldsymbol{\gamma}_{\boldsymbol{a} 0}\right)$ & 0.04 & $0.01-0.06$ \\
Alcohol use $\rightarrow$ risk-taking $\left(\boldsymbol{\gamma}_{\boldsymbol{b 0}}\right)$ & 2.31 & $1.72-2.81$ \\
Sensation seeking $\rightarrow$ risk-taking $\left(\boldsymbol{\gamma}_{\boldsymbol{c}, \mathbf{0}}\right)$ & 0.46 & $0.09-0.84$ \\
Covariance $\left(\boldsymbol{r}_{\boldsymbol{u a u b}}\right)$ & 0.01 & $-0.03-0.06$ \\
Indirect effect & 0.11 & $0.02-0.18$ \\
Total effect & 0.57 & $0.19-0.94$ \\
\hline
\end{tabular}

Notes: $N=2737$ days nested within 167 participants. 


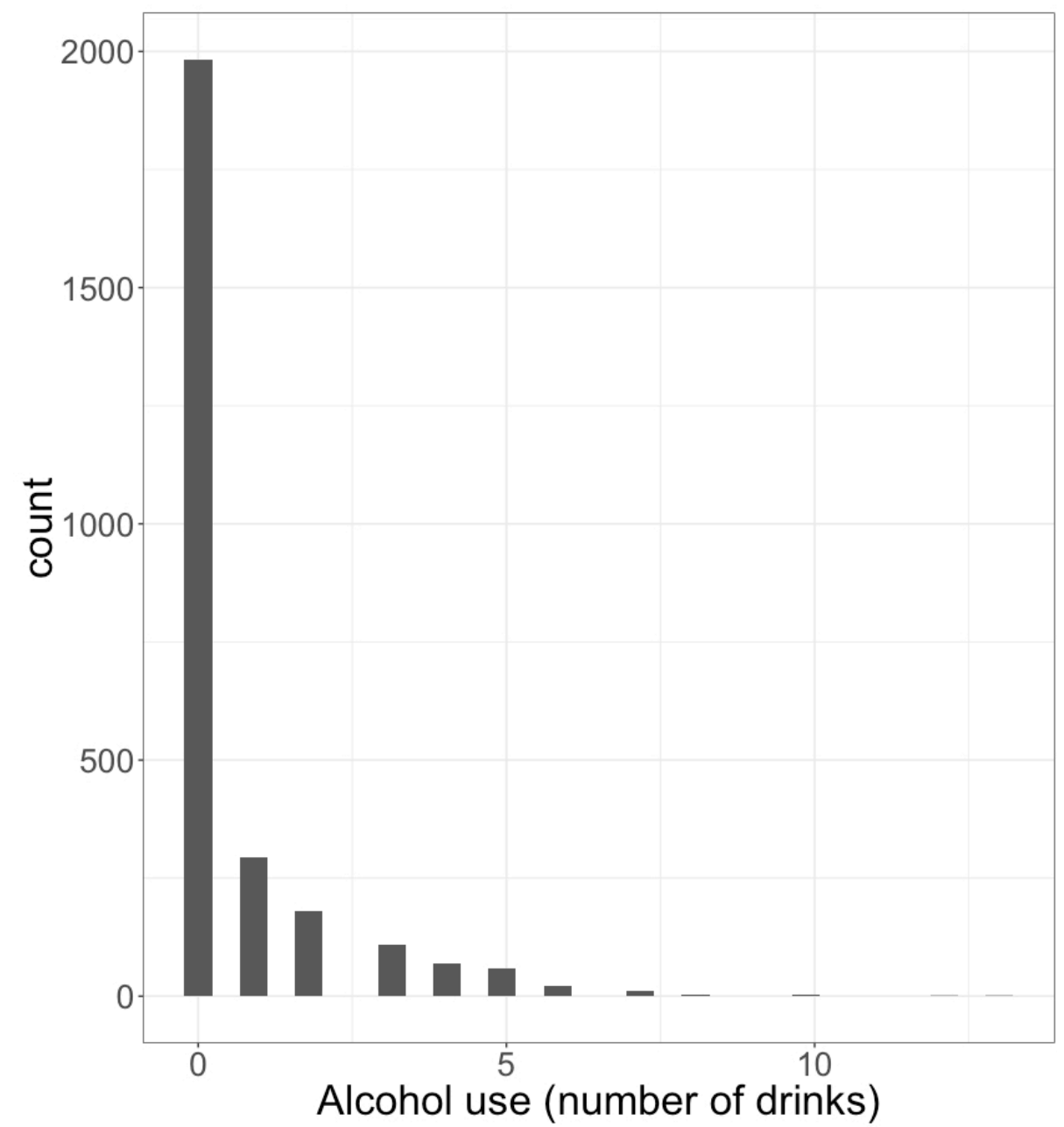

Figure S1. Distribution of the alcohol use variable. The count (y-axis) of number of alcohol drunks consumed (x-axis) each day is positively skewed and has many zero entries, motivating the use of a hurdle model in the present study. 


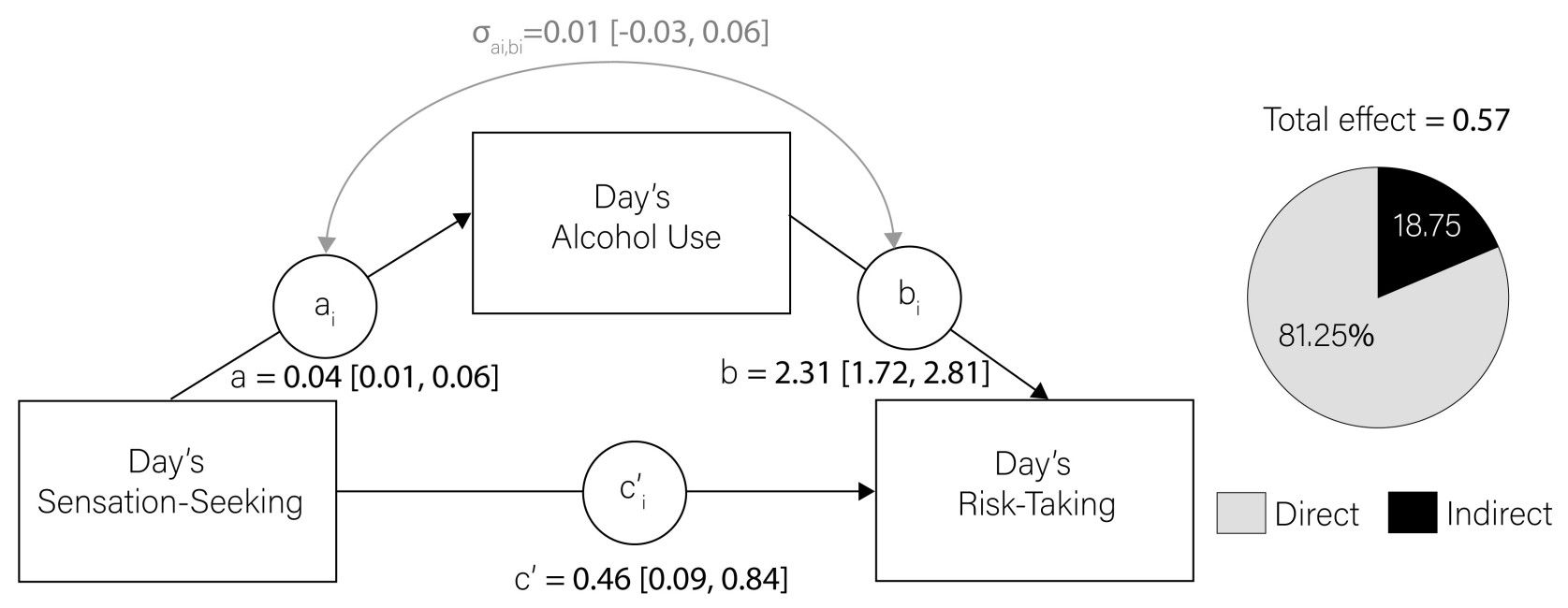

Figure S2. Results of the within-person mediation analysis. We used multilevel mediation (Bauer et al., 2006) using a convenience function available at http://www.pagegould.com/r/indirectmlm/ that makes use of the boot package in R (Canty \& Ripley, 2017) to examine the within-person association between sensation-seeking, alcohol use, and risk-taking. All variables consisted of time-varying components where 0 indicates a day of average levels of that variable for each person, values above zero indicate higher than usual levels, and values below zero indicate lower than usual levels (Bolger \& Laurenceau, 2013). Days of higher than usual sensation-seeking were associated with higher than usual alcohol use $(a)$ and days of higher than usual alcohol use were associated with days of higher than usual risk-taking $(b)$. The direct effect of day's sensation-seeking on risk-taking $\left(c^{\prime}\right)$ was significant after inclusion of the indirect path to risk-taking via increases in alcohol use. The pie chart illustrates the portion of the effect of day's sensation-seeking on risk-taking accounted for by alcohol use. 


\section{References}

Bauer, D.J., Preacher, K.J., \& Gil, K.M. (2006). Conceptualizing and testing random indirect effects and moderated mediation in multilevel models: New procedures and recommendations. Psychological Methods, 11(2), 142-163

Bolger, N., \& Laurenceau, J. P. (2013). Intensive longitudinal methods. New York, NY: Guilford. Canty, A., \& Ripley, B. (2017). boot: Bootstrap R (S-Plus) functions. R package version 1.3-20. 